\section{IN THE NEWS}

Reverting to type

Haiti and the Dominican

Republic, despite being

declared polio-free in the

1980s, suffered from a

surprise oubreak of

poliomyelitis in 2000. Twenty-

one children were infected

and two of these cases were

fatal. A report published

on 14 March 2002 in

Sciencexpress (the on-line,

early edition of the journal

Science) that investigated

possible causes of the

resurgence of this crippling

disease has hit the headlines

this month.

In these countries, an

oral polio vaccine (OPV),

which contains a live but

attenuated form of

poliovirus, is used to protect

people from infection.

As detailed in the report, and

explained by Olen Kew of the

US Centers for Disease

Control and Prevention,

Atlanta, Georgia, "In Haiti,

after the wild-type poliovirus

had been eradicated, the

only source was through the

polio vaccine." (Associated

Press).

As summed up in an article

from Reuters, genetic

analysis of viral samples from

the outbreak confirmed that

the source of infection was

in fact the vaccine virus itself,

which had mutated back to

a virulent form by swapping

DNA with related viruses,

known as enteroviruses, that

are present in the gut.

The situation might have

been exacerbated by the low

rates of vaccination in Haiti.

"Low population immunity

was the key risk factor,"

explained Kew.

When a person is

vaccinated with OPV, the

vaccine virus can mutate, be

shed in faeces, spread to

non-immunized individuals in

the water supply and cause

disease. "It's a warning that

you need to have good

coverage to prevent vaccines

from running away like this," said Kew (New Scientist)

These results have obvious

implications for the aims of

the World Health

Organisation to eradicate polio worldwide.

Jenny Buckland

\title{
A balanced response
}

For many decisions in life, it is important to weigh up different factors before making a decision as to the best course of action. A recent Nature paper from Jason Cyster's lab now shows that this is also true for B cells antigen-stimulated B-cell migration seems to be governed by the balance of responses to the T-cell-zone chemokines CCL19 and CCL21, and the B-cell-zone chemokine CXCL13.

$B$ cells scan for antigen by recirculating between the B-cell follicles of secondary lymphoid organs. When they encounter antigen, B cells in the B-cell zone or follicle migrate to the boundary between the T-cell and B-cell zones ( $\mathrm{T} / \mathrm{B}$ boundary), where they interact with $\mathrm{T}$ cells. However, the factors that control this migration are undefined. Genetic studies have shown that CCR7 and its ligands, CCL19 and CCL21, are important for the localization of $\mathrm{T}$ cells and dendritic cells to the T-cell zone. Cyster and colleagues tested whether CCR7 is also important for B-cell localization. Anti-IgMstimulated B cells had a 2-3-fold increased expression of CCR7 and enhanced ex vivo chemotactic responses to CCL19 and CCL21. When transferred into new hosts, the stimulated B cells localized to the T/B boundary.

The authors wanted to test the idea that antigenmediated relocalization of $\mathrm{B}$ cells in vivo depends on the expression of CCR7 and responsiveness to CCL19 and CCL21. They transferred hen egg lysozyme (HEL)specific transgenic B cells into CCL19-/- CCL2 $1^{-/-}$ recipients. In the absence of antigen stimulation, the $B$ cells localized in the follicles, but antigen stimulation did not induce migration to the $\mathrm{T} / \mathrm{B}$ boundary. Because these results might have been due to indirect effects downstream of T-cell-zone chemokine deficiency, similar experiments were then performed on a CCR7-deficient background, to test for an intrinsic requirement for CCR7. In CCR7-deficient mice, HELbinding $\mathrm{B}$ cells did not localize to the T/B boundary, but actually migrated in the opposite direction to that observed in wild-type mice, so that the cells localized in the pole of the follicle distal to the T-cell zone. So, CCL19 and CCL21 are important for attracting antigen-stimulated $\mathrm{B}$ cells towards the T-cell zone, but why do the $B$ cells only travel as far as the T/B boundary? The authors tested whether responsiveness to the B-cell-zone chemokine CXCL13 might limit the migration of B cells into the T-cell zones. Retroviral transduction was used to upregulate the expression of CCR7 on B cells, which were then transferred into new hosts. Transduced wild-type B cells migrated to the T/B boundary as expected, but transduced cells that were deficient for CXCR5 - the receptor for CXCL13 migrated fully into the $\mathrm{T}$-cell zone and did not line up along the $\mathrm{T} / \mathrm{B}$ boundary.

These results show that the precise localization of antigen-stimulated B cells in secondary lymphoid tissue is dependent on the balance of responsiveness

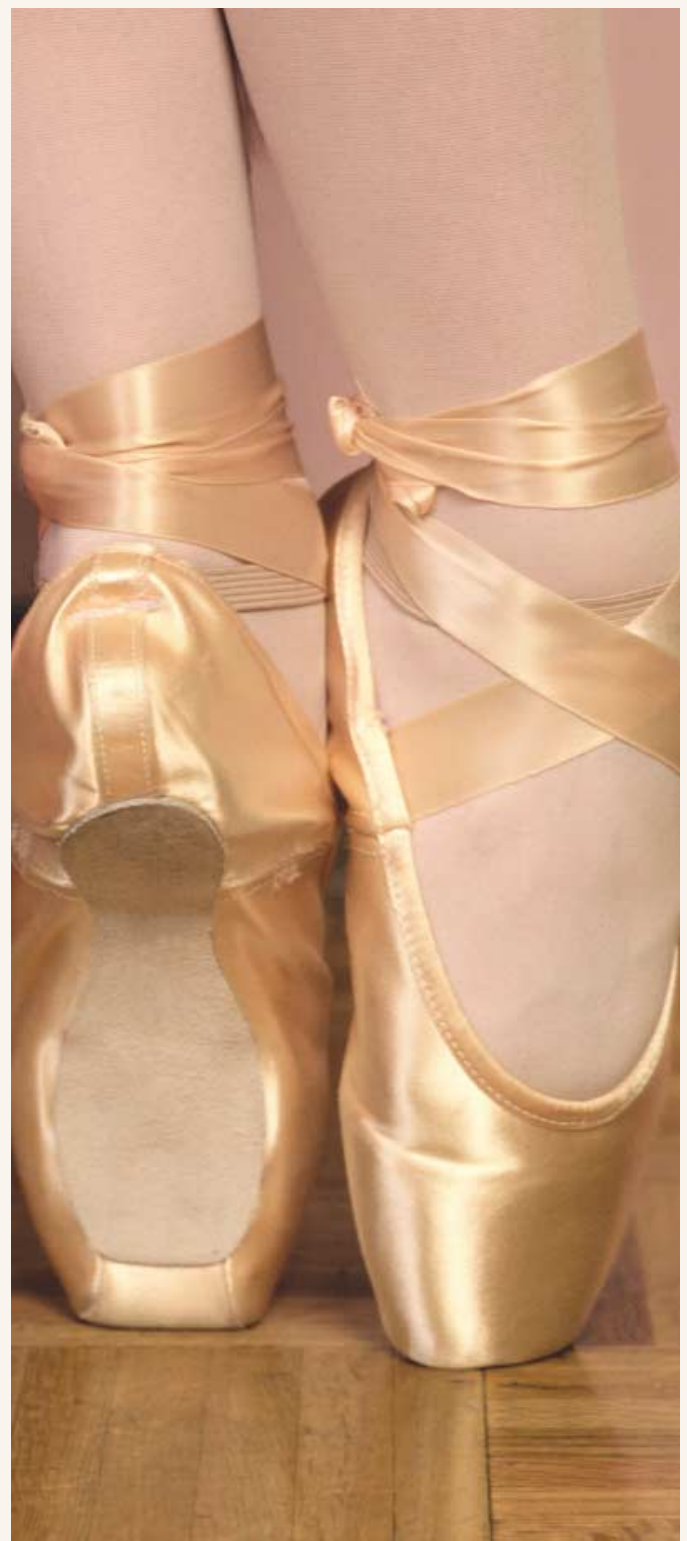

to chemokines that are generated in adjacent zones CCL19 and CCL21, which attract B cells to the T-cell zone, and CXCL13, which attracts B cells to the B-cell zone.

Elaine Bell

(Q) References and links

ORIGINAL RESEARCH PAPER Reif, K. et al. Balanced responsiveness to chemoattractants from adjacent zones determines B-cell position. Nature 416, 94-99 (2002)

FURTHeR READING Kunkel, E. J. \& Butcher, E. C. Chemokines and the tissue-specific migration of lymphocytes. Immunity 16, 1-4 (2002) WEB SITES

Jason Cyster's lab:

http://itsa.ucsf.edu/ micro/Faculty/CysterSite/home.htm

Encyclopedia of Life Sciences: http://www.els.net/ chemokines 